\title{
ANALYTIC ITERATION(1)
}

BY

\author{
ERI JABOTINSKY
}

1. Introduction. Definitions. Plan

Let $\Omega$ be a family of analytic functions such that $F(z) \in \Omega$ if and only if:

$$
F(z)=\sum_{k=1}^{k=\infty} f_{k} z^{k}, \text { with } f_{1}=1 \text {, for }|z|<\rho_{F}, \rho_{F}>0 .
$$

In the present paper we propose to study the iterates of functions $F(z) \in \Omega$. We define here the $s$-iterate of the function $F(z) \in \Omega$ as in a joint paper with P. Erdös [3]:

Let $S$ be $a$ set of complex numbers such that $a \in S$ and $b_{.} \in S$ implies $(a-b) \in S$. The function $F(z) \in \Omega$ will be said to possess iterates in $S$ if there exists a function $F(s, z)$, called the s-iterate of $F(z)$, defined for $s \in S$ and satisfying the following four conditions:

$$
F(1, z)=F(z) .
$$

$$
\text { Considered as a function of } z, F(s, z) \in \Omega \text { for every } s \in S \text {. }
$$

$$
\begin{gathered}
F\left[s, F\left(s^{\prime}, z\right)\right]=F\left[\left(s+s^{\prime}\right), z\right], \quad s, s^{\prime} \in S . \\
F(s, z)=\sum_{k=1}^{k=\infty} f_{k}(s) z^{k}\left(s \in S,|z|<\rho_{F, s}, \rho_{F, s}>0\right), \\
\text { where the } f_{k}(s) \text { are polynomials in } s .
\end{gathered}
$$

If $S$ is the set of all integers, $F(s, z)$ is said to be the integer iterate of $F(z)$. If $S$ is the set of all (finite) real numbers, $F(s, z)$ is the complete real iterate of $F(z)$. If $S$ is the set of all (finite) complex numbers, $F(s, z)$ is said to be the complete complex iterate of $F(z)$.

It was shown in [3] that in every case $F(s, z)$ is completely defined by the four conditions (1.1), (1.2), (1.3) and (1.4). It was also shown there that if $F(s, z)$ is the complete real iterate of $F(z)$, then it is also the complete complex iterate of $F(z)$ and that, moreover, for every finite complex $s_{0}$ there exists a $\rho_{s_{0}}>0$ such that if $|z|<\rho_{s_{0}}$ then $F(s, z)$, considered as a function of $s$, is analytic in $s$ in some neighborhood of $s$. (This result is, in effect, a sharper form of a theorem of $\mathrm{S}$.

Received by the editors August 1, 1962.

(1) The research reported in this document has been sponsored by the Air Force Office of Scientific Research of the Air Research and Development Command, United States Air Force, through its European Office. Contract No. AF 61(052)-482. 
Bochner and D. Montgomery [2] on transformation groups in the special case of iteration.) In this case $F(s, z)$ is said to be the analytic iterate of $F(z)$. Hence: every complete real iterate is an analytic iterate.

We shall be concerned, in this paper, with the power series expansions of various powers of the $s$-iterates $F(s, z)$. We shall write for any complex $t$ :

$$
\left(\frac{F(s, z)}{z}\right)^{t}=\sum_{k=0}^{k=\infty} f(s, t, k) z^{k} \text {. }
$$

We shall consider that branch of this function which $=1$ for $z=0$ so that we take:

$$
f(s, t, 0)=1 \text {. }
$$

Comparing (1.4) and (1.5) we find that:

$$
f_{k}(s)=f(s, 1, k-1) .
$$

A further form of notation will be used in the case that $t$ is an integer (negative, zero or positive). Putting $t=m$ we shall then write:

so that:

$$
(F(s, z))^{m}=\sum_{k=m}^{k=\infty} f_{m, k}(s) z^{k}
$$

$$
f_{m, k}(s)=f(s, m, k-m) \text { and } f_{1, k}(s)=f_{k}(s) .
$$

Our main purpose is to obtain explicit expressions for the coefficients $f(s, t, k)$ in (1.5). Two principal tools will be used:

(A) The representation of functions by matrices.

(B) The $L$-sequences and the $L$-functions.

The representation of functions by matrices will be defined, together with some of its properties, in $\$ 2$. These will be used in $\$ 3$ to prove the classical result that, for integer $s$, the coefficients $f_{k}(s)$ in (1.4) are uniquely defined and are polynomials in $s$ of degree not exceeding $(k-1)$. Similar results will be obtained for the coefficients $f_{m, k}(s)$ in (1.8).

In $\S 3$ we introduce the $L$-sequences $\left\{l_{j}\right\}$ by the defining relation $l_{j}=f_{j+1}^{\prime}(0)$ (the differentiation is with respect to $s$ ). In the particular case when $F(s, z)$ is an analytic iterate we shall define the $L$-sequences by the equivalent definitions:

$$
L(z)=\left.\frac{\partial F(s, z)}{\partial s}\right|_{s=0} \text { and } L(z)=\sum_{j=1}^{j=\infty} l_{j} z^{j+1} .
$$

(It was shown in [3] that when $F(z)$ has no analytic iterate the last series diverges for every $z \neq 0$.)

In $\S 4$ the $L$-sequences are used to give an explicit expression for the coefficients $f(s, t, k)$ of $(1.5)$ for all complex $t$ and all $s \in S$. In $\$ 5$ Grunsky's coefficients are expressed in terms of $\left\{l_{j}\right\}$.

Added in proof. In the last paragraphs these results are applied to various problems. 


\section{REPRESENTATION OF FUNCTIONS BY MATRICES}

Let $F(z) \in \Omega$ and let $m$ be an integer (negative, zero or positive). We expand $(F(z))^{m}$ in a power series in $z$ and write:

$$
(F(z))^{m}=\sum_{k=m}^{k=+\infty} f_{m, k} z^{k}, \text { for }|z|<\rho, \rho>0 .
$$

(There may be a different radius of convergence for $m<0$ and for $m>0$. In this case we take $\rho$ to be the smallest of the two.) If we put:

$$
f_{m k}=0 \text { for } m>k,
$$

we can rewrite $(2.1)$ in the form:

$$
(F(z))^{m}=\sum_{k=-\infty}^{k=+\infty} f_{m, k} z^{k}, \text { for }|z|<\rho, \rho>0 .
$$

In this manner we have obtained from the function $F(z)$ the doubly infinite matrix :

$$
f=\left\|f_{m, k}\right\|, \quad(-\infty<m, k<+\infty),
$$

whose elements $f_{m . k}$ are defined by (2.1) and by (2.2).

The matrix $f$ is said to represent the function $F(z)$ and will be called a representation matrix.

It might be noted that the functions $F(z) \in \Omega$ form a group $\Gamma$ if we take as a group product of the two functions $F_{1}(z)$ and $F_{2}(z)$ the function $F_{1}\left(F_{2}(z)\right)$. The neutral element of the group $\Gamma$ is the identical function $z$. The representation matrices will now be shown to form a group (with the usual matrix product as group product) which is isomorphic to $\Gamma$. The representation matrices therefore constitute a representation of $\Gamma$ in the usual sense.

The main interest of the representation matrices results precisely from the existence of this isomorphism. The corresponding property can be stated as follows:

Consider the two functions $F(z) \in \Omega$ and $G(z) \in \Omega$ and the function $K(z)=F(G(z))$ which is also $\in \Omega$. Let $f, g$ and $k$ be the matrices representing these functions. Then:

$$
k=f \times g, \text { (matrix multiplication). }
$$

That is:

$$
k_{m, n}=\sum_{p=-\infty}^{p=+\infty} f_{m, p} g_{p, n}
$$

(Note that there are only a finite number of nonzero terms in this sum: those for which $m<p<n$.)

The proof is uy writing $(F(z))^{m}=\sum_{p=-\infty}^{p=+\infty} f_{m, p} z^{p}$ and by substituting the expansion $(G(z))^{p}=\sum_{n=-\infty}^{n=+\infty} g_{p, n} z^{n}$ for $z^{p}$ and rearranging the terms, which is legitimate. 
We now apply the above result to obtain explicit expressions for the coefficients $f_{m, k}(s)$ and $f_{k}(s)$ in (1.8) and (1.4) for the case of integer $s$.

Let $s$ be a positive integer. Then, by the repeated application of (2.5), we see that the matrix representing the s-iterate $F(s, z)$ of the function $F(z) \in \Omega$ is the sth power of the matrix $f$ representing $F(z)$. We denote this matrix by $f^{s}$.

Let $\delta$ be the unit matrix:

$$
\delta=\left\|\delta_{m, n}\right\|, \quad(-\infty<m, n<+\infty) .
$$

This matrix commutes with all matrices. It is also the representation matrix of the identical function $z$. As the identical function $z$ can be considered to be the 0 -iterate of any function $F(z)$, we shall write:

$$
f^{0}=\delta \text {. }
$$

Now put

$$
\hat{f}=f-\delta \quad \text { or: } \quad f=\hat{f}+\delta .
$$

Then, because $\delta$ commutes with all matrices, we have, using (2.8):

$$
f^{s}=(\hat{f}+\delta)^{s}=\sum_{q=0}^{q=s}\left(\begin{array}{l}
s \\
q
\end{array}\right) \hat{f}^{q} .
$$

Denoting by $\left(f^{s}\right)_{m, k}$ and by $\left(\hat{f}^{q}\right)_{m, k}$ the $m, k$ element of the matrices $f^{s}$ and $\hat{f}^{q}$ respectively, we get:

$$
\left(f^{s}\right)_{m, k}=\sum_{q=0}^{q=s}\left(\begin{array}{l}
s \\
q
\end{array}\right)\left(\hat{f}^{q}\right)_{m, k}
$$

But by (2.2) and (2.9) we have $\hat{f}_{m, k}=0$ for $m>k-1$ and hence:

$$
\left(\hat{f}^{q}\right)_{m, k}=0 \text { for } m>k-q \text {, that is, for } q>k-m \text {. }
$$

It follows from (2.12) that in the sum (2.11) only terms with $q \leqq k-m$ are $\neq 0$, so that $(2.11)$ may be written:

$$
\left(f^{s}\right)_{m, k}=\sum_{q=0}^{q=k-m}\left(\hat{f}^{q}\right)_{m, k}\left(\begin{array}{l}
s \\
q
\end{array}\right) .
$$

But $\left(\begin{array}{l}s \\ q\end{array}\right)$ is a polynomial in $s$ of degree $q$ and the numbers $\left(\hat{f}^{q}\right)_{m, \tilde{k}}$ are independent of $s$. Therefore the right side of (2.13) is a polynomial in $s$ of degree not higher than $(k-m)$. As $f^{s}$ is the representation matrix of $F(s, z)$ we have for any integer $m$ :

$$
[F(s, z)]^{m}=\sum_{k=-\infty}^{k=+\infty}\left({ }^{s}\right)_{m, k} z^{k}=\sum_{k=-\infty}^{k=+\infty}\left[\sum_{q=0}^{q=k-m}\left(\hat{f}^{q}\right)_{k, m}\left(\begin{array}{l}
s \\
q
\end{array}\right)\right] z^{k},
$$

and in the particular case when $m=1$ : 


$$
F(s, z)=\sum_{k=-\infty}^{k=+\infty}\left(f^{s}\right)_{1, k} z^{k}=\sum_{k=-\infty}^{k=+\infty}\left[\sum_{q=0}^{q=k-1}\left(f^{q}\right)_{k, 1}\left(\begin{array}{l}
s \\
q
\end{array}\right)\right] z
$$

Comparing (2.14) and (2.15) respectively with (1.8) and (1.4), we note that $\left(f^{s}\right)_{m, k}=f_{m, k}(s)$ and $\left(f^{s}\right)_{1, k}=f_{k}(s)$. Therefore:

$$
f_{m, k}(s)=\sum_{q=0}^{q=k-m}\left(\hat{f}^{q}\right)_{m, k}\left(\begin{array}{l}
s \\
q
\end{array}\right)
$$

and:

$$
f_{k}(s)=\sum_{q=0}^{q=k-1}\left(\hat{f}^{q}\right)_{1, k}\left(\begin{array}{l}
s \\
q
\end{array}\right)
$$

These are the desired expressions for $f_{m, k}(s)$ and for $f_{k}(s)$, which are seen to be polynomials in $s$ of degree not exceeding $(k-m)$ and $(k-1)$ respectively. The above proof of this well-known result follows [6] except that here use was made of the full representation matrix $\left\|f_{m, n}\right\|$ with $-\infty<m, n<+\infty$, while in [6] only matrices with $0<m, n<+\infty$ were used.

Before proceeding it may be of independent interest to express the coefficient $f_{m, k}(s)$ and $f_{k}(s)$ in terms of the matrices $f$ (instead of the matrices $\hat{f}$ ). For this purpose, using (2.9) we substitute $(f-\delta)$ for $\hat{f}$ in (2.16) and (2.17). After some computation, using usual combinatorial formulas, we get:

$$
f_{m, k}(s)=\sum_{q=0}^{q=k-m}(-1)^{k-m-q}\left(f^{q}\right)_{m, k}\left(\begin{array}{l}
s-1-q \\
k-m-q
\end{array}\right)\left(\begin{array}{l}
s \\
q
\end{array}\right),
$$

and:

$$
f_{k}(s)=\sum_{q=0}^{q=k-1}(-1)^{k-1-q}\left(f^{q}\right)_{1, k} \quad\left(\begin{array}{l}
s-1-q \\
k-1-q
\end{array}\right)\left(\begin{array}{l}
s \\
q
\end{array}\right) .
$$

Formulas (2.16) and (2.17), or their equivalents (2.18) and (2.19), were established for positive integer $s$ only, but their right sides preserve a meaning for all complex $s$. The series (2.15) for positive integer $s$ has a positive radius of convergence and its sum, being $F(s, z)$, satisfies the four conditions (1.1) to (1.4). Therefore, substituting this series into both sides of (1.3) we must find that, for positive integer values of $s$ and $s^{\prime}$, equal powers of $z$ have equal coefficients on both sides. But these coefficients are polynomials in $s$ and $s^{\prime}$ and two polynomials in $s$ and $s^{\prime}$ which are numerically equal for all positive integer values of $s$ and of $s^{\prime}$, are identical. We have thus the result:

If the series (2.15) for $F(s, z)$ has, considered as a function of $z$, a positive radius of convergence for all given complex $s$, then it satisfies the four conditions (1.1) to (1.4) for all complex $s$ and $s^{\prime}$ and its sum is the analytic iterate $F(s, z)$ of $F(z)$.

It is easily seen that the radius of convergence of the series (2.15) is always 
positive for integer $s$ (negative, zero or positive). Therefore the sum of this series gives the integer iterate of every function $F(z) \in \Omega$.

\section{3. $L$-SEQUENCES AND $L$-FUNCTIONS}

Consider the derivatives $f_{k}^{\prime}(s)$ of the polynomials $f_{k}(s)$ constructed in the previous section. These polynomials, and therefore their derivatives, exist whether the series in (2.15) has or has not a positive radius of convergence. The numbers $l_{j}$, defined by:

$$
l_{j}=f_{j+1}^{\prime}(0),
$$

exist and are uniquely determined by the functions $F(z) \in \Omega$. (Note that we could have written $l_{j+1}$ instead of $l_{j}$, but, as will become apparent in the next section, the notation of (3.1) is more economic.)

The values of the numbers $l_{j}$ may be obtained by noting that:

$$
\left(\begin{array}{l}
0 \\
n
\end{array}\right)=\delta_{0 . n} \text { and }\left.\frac{d}{d s}\left(\begin{array}{l}
s \\
n
\end{array}\right)\right|_{s=0}=\frac{(-1)^{n-1}}{n} \text {. }
$$

And hence, from (2.17) and from (2.19) we obtain $l_{j}$ in terms of the elements of the powers of the matrices $\hat{f}$ and $f$ respectively. We find:

$$
l_{j}=\sum_{q=1}^{q=j} \frac{(-1)^{q+1}}{q}\left(\hat{f}^{q}\right)_{1, j+1},
$$

and:

$$
l_{j}=\sum_{q=0}^{q=j} \frac{(-1)^{q+1}}{q}\left(\begin{array}{l}
j \\
q
\end{array}\right)\left(f^{q}\right)_{1, j+1} .
$$

In the particular case when $F(z)$ has an analytic iterate we can generate the $l_{j}$ by differentiating equation (1.4) with respect to $s$ and putting $s=0$ in the result. The series obtained on the right has in this case a positive radius of convergence and defines an analytic function which we denote by $L(z)$. We have then:

$$
L(z)=\left.\frac{\partial F(s, z)}{\partial s}\right|_{s=0}=\sum_{j=1}^{j=+\infty} l_{j} z^{j+1}
$$

Moreover, in this case, we may differentiate equation (1.3) both with respect to $s$ and with respect to $s^{\prime}$. Differentiating with respect to $s$ we obtain:

$$
\frac{\partial F\left[s, F\left(s^{\prime}, z\right)\right]}{\partial s}=\frac{\partial F\left[\left(s+s^{\prime}\right), z\right]}{\partial\left(s+s^{\prime}\right)} \cdot \frac{\partial\left(s+s^{\prime}\right)}{\partial s} \text {. }
$$

Putting $s=0$ in (3.6) and using (3.5) we get:

$$
L\left(F\left(s^{\prime}, z\right)\right)=\frac{\partial F\left(s^{\prime}, z\right)}{\partial s^{\prime}} .
$$


Differentiating now (1.3) with respect to $s^{\prime}$ we obtain:

$$
\frac{\partial F\left[s, F\left(s^{\prime}, z\right)\right]}{\partial F\left(s^{\prime}, z\right)} \cdot \frac{\partial F\left(s^{\prime}, z\right)}{\partial s^{\prime}}=\frac{\partial F\left[\left(s+s^{\prime}\right), z\right]}{\partial\left(s+s^{\prime}\right)} \cdot \frac{\partial\left(s+s^{\prime}\right)}{\partial s^{\prime}} .
$$

Putting now $s^{\prime}=0$ in (3.8) and using (3.5) and the fact that $F(0, z)=z$ we get:

$$
\frac{\partial F(s, z)}{\partial z} \cdot L(z)=\frac{\partial F(s, z)}{\partial s} .
$$

Changing $s^{\prime}$ into $s$ in (3.7) and combining with (3.9) we finally obtain the multiple equation [9]:

$$
\frac{\partial F(s, z)}{\partial s}=L(F(s, z))=\frac{\partial F(s, z)}{\partial z} \cdot L(z)=\frac{\partial F(s, z)}{\partial s} .
$$

This double equation is fundamental in the theory of iteration. It can be used and extended in many ways.

Thus, from the first equality of (3.10), by further differentiation, first with respect to $s$ and then with respect to $z$, we easily obtain after eliminating $L^{\prime}(F(s, z))$, a partial differential equation [5] satisfied by all analytic iterates (actually the validity of this partial differential equation is much larger):

$$
\frac{\partial^{2} F}{\partial s^{2}} \cdot \frac{\partial F}{\partial z}=\frac{\partial^{2} F}{\partial s \cdot \partial z} \cdot \frac{\partial F}{\partial s}
$$

From the second equality of (3.10) we obtain an ordinary differential equation satisfied by analytic iterates [3]. Indeed put $w=F(s, z)$. We find, for fixed $s$ :

$$
\frac{d w}{d z}=\frac{L(w)}{L(z)}
$$

Finally the third equality of (3.10) can be made to yield a more general equation. Indeed let $t$ be any complex number and let $(F(s, z))^{t}$ denote any given branch of that function. Then, multiplying the first and the last term of (3.10) by $t(F(s, z))^{t-1}$, we find:

$$
\frac{\partial(F(s, z))^{t}}{\partial s}=\frac{\partial(F(s, z))^{t}}{\partial z} \cdot L(z)
$$

Equation (3.13) will be extensively used in the sequel so that we are interested in extending its validity outside the domain of analytic iterates (for which it was established).

Specifically: if in (3.13) we substitute for $(F(s, z))^{t}$ and for $L(z)$ their power series (1.5) and (3.5) respectively and carry out formally the operations of partial differentiation and of multiplication the coefficients of equal power of $z$ on 
both sides of (3.13) will be equal even if $L(z)$ has a zero radius of convergence and even if $s$ is given a value not in $S$, so that $F(s, z)$ too has a zero radius of convergence.

This could be shown by rewording the argument leading to (3.13) in terms of the Calculus of Formal Series. However, the following argument may be shorter: Let us replace the sequence $L=\left\{l_{j}\right\}$ by the sequence $L^{*}=\left\{l_{j}^{*}\right\}$ where $l_{j}^{*}=l_{j}$ for $1 \leqq j \leqq n$ and $l_{j}^{*}=0$ for $j>n$. The function $L^{*}(z)=\sum_{j=1}^{j=\infty} l_{j}^{*} z^{j+1}$ is then a polynomial so that the corresponding function $F^{*}(z)$ has an analytic iterate (by a result of [3]), so that (3.13) holds for the functions $L^{*}(z)$ and $F^{*}(s, z)$ for all complex $t$ and $s$ and sufficiently small $z$. It follows that (3.13) also holds formally for the expansions of these functions. But the first $n$ terms of the expansions in power series in $z$ of $L^{*}(z)$ and of $F^{*}(s, z)$ coincide with the corresponding $n$ terms of the expansion of $L(z)$ and $F(s, z)$. As $n$ is arbitrary we have proven that (3.13) holds formally for the expansions(1.5) and (3.5) of $F(s, z)$ and $L(z)$.

Note that the function $L(z)$ is the result of applying a certain operator to the function $F(z)$. In the author's thesis [9] the use of the following notation was suggested:

$$
L(z)=\operatorname{LOG} F(z) .
$$

This is justified by comparing the properties of the $L$-function with those of the usual logarithm and becomes even more apparent if we denote the s-iterate of $F(z)$ by $F^{s}(z)$. Thus we have:

$$
\left.\frac{d\left(f^{s}\right)}{d s}\right|_{s=0}=\log f \quad \text { and }\left.\quad \frac{\partial F^{s}(z)}{\partial s}\right|_{s=0}=\operatorname{LOG} F(z) .
$$

Also, as is easily verified:

$$
\log f^{a}=a \log f \quad \text { and } \quad \operatorname{LOG} F^{a}(z)=a \operatorname{LOG} F(z) .
$$

Furthermore, if $F(z) \in \Omega$ and $G(z) \in \Omega$ and if $F[G(z)]=G[F(z)]$ it can be shown [5] that $G(z)$ is some iterate of $F(z)$ and hence that:

$$
\operatorname{LOG} F[G(z)]=\operatorname{LOG} G[F(z)]=\operatorname{LOG} F(z)+\operatorname{LOG} G(z) .
$$

In spite of these analogies, the notation is cumbersome and has been abandoned.

Finally, from (3.12) it follows that the $L$-function is related to quadratic differentials [12].

\section{MAIN THEOREM}

Explicit expansion of $f(t, k, s)$ in terms of the $L$-sequence.

The formalinterpretation of equation (3.13) enables us now to prove the main theorem of this paper:

THEOREM I. 1. The sequence $\left\{f_{1}=1, f_{2}, f_{3}, \cdots\right\}$ determines uniquely the sequence $\left\{l_{1}, l_{2}, \cdots\right\}$ through the equations: 


$$
f_{k}=\sum \frac{1}{r !} k_{0} k_{1} \cdots k_{r-1} l_{d_{1}} l_{d_{2}} \cdots l_{d_{r}},
$$

the sum being taken for all integer $r$ and all integer $k_{i}$ such that:

$$
1=k_{0}<k_{1}<\cdots<k_{r-1}<k_{r}=k \text { and } d_{i}=k_{i}-k_{i-1} .
$$

2. Let $t$ be any finite complex number. Let $s \in S$ be a complex number such that $F(s, z)$ is the s-iterate of the function $F(z) \in \Omega$ in the sense of $\S 1$. Put:

$$
(F(s, z))^{t}=z^{t} \sum_{k=0}^{k=+\infty} f(s, t, k) z^{k}
$$

Then:

$$
f(s, t, 0)=1
$$

and:

$$
f(s, t, k)=\sum \frac{s^{r}}{r !}\left(t+k_{0}\right)\left(t+k_{1}\right) \cdots\left(t+k_{r-1}\right) l_{d_{1}} l_{d_{2}} \cdots l_{d_{r}}, \quad(k>0),
$$

the sum being taken for all integer $r$ and all integer $k_{i}$ such that:

$$
0=k_{0}<k_{1}<\cdots<k_{r-1}<k_{r}=k \text { and } d_{i}=k_{i}-k_{i-1} .
$$

Proof. Equation (4.1') is seen to be of the form $f_{k}=l_{k-1}+\phi\left(l_{1}, l_{2}, \cdots, l_{k-2}\right)$, so that, given the sequence $\left\{f_{i}\right\}$, the terms of the sequence $\left\{l_{j}\right\}$ can all be determined successively. Equation (4.2") is just (1.6). From this and from (4.2"') it follows that:

$$
f(0, t, k)=\delta_{0, k} .
$$

Let us write:

$$
\frac{\partial}{\partial s} f(s, t, k)=f^{\prime}(s, t, k) .
$$

Then from (4.2) it follows that, formally:

$$
\frac{\partial(F(s, z))^{t}}{\partial s}=z^{t} \sum_{k=0}^{k=+\infty} f^{\prime}(s, t, k) z^{k},
$$

and:

$$
\frac{\partial(F(s, z))^{t}}{\partial z}=\sum_{k=0}^{k=+\infty}(t+k) f(s, t, k) z^{t+k-1}
$$

Define the sequence $\left\{l_{k}^{\prime}\right\}$ by:

$$
\left.\frac{\partial F(s, z)}{\partial s}\right|_{s=0}=\sum_{k=1}^{k=+\infty} l_{k}^{\prime} z^{k+1} .
$$


Then (3.13) becomes:

$$
\sum_{k=0}^{k=+\infty} f^{\prime}(s, t, k) z^{t+k}=\left[\sum_{k=1}^{k=+\infty} l_{k}^{\prime} z^{k+1}\right]\left[\sum_{k=0}^{k=+\infty}(t+k) z^{t+k-1}\right] .
$$

Equating the coefficients of $z^{t+k}$ on both sides of (4.8) we find that:

$$
f^{\prime}(s, t, k)=\sum_{q=0}^{q=k-1}(t+q) l_{k-q}^{\prime} f(s, t, q)
$$

We shall now prove by induction that, more generally:

$$
\frac{\partial^{r}}{\partial s^{r}} f(s, t, k)=\sum\left(t+k_{0}\right)\left(t+k_{1}\right) \cdots\left(t+k_{r-1}\right) l_{d_{1}} l_{d_{2}} \cdots l_{d} \cdot f\left(t, k_{0}, s\right),
$$

the sum being taken for all integer $k_{i}$ such that:

$$
0 \leqq k_{0}<k_{1}<\cdots<k_{r-1}<k_{r}=k \text { and } \quad d_{i}=k_{i}-k_{i-1} .
$$

Indeed, from (4.9) we see that (4.10) holds for $r=1$. We now suppose that (4.10) holds for $r$. Differentiating both sides of (4.10) with respect to $s$ and using (4.9) on the right-hand side we find that:

$$
\begin{aligned}
\frac{\partial^{r+1}}{\partial s^{r+1}} f(s, t, k) & =\sum\left(t+k_{0}\right)\left(t+k_{1}\right) \cdots\left(t+k_{r-1}\right) l_{d_{1}}^{\prime} l_{d_{2}}^{\prime} \cdots l_{d}^{\prime} f^{\prime}\left(s, t, k_{0}\right) \\
& =\sum\left(t+k_{0}\right)\left(t+k_{1}\right) \cdots\left(t+k_{r-1}\right) l_{d_{1}}^{\prime} l_{d_{2}}^{\prime} \cdots l_{d}^{\prime}\left(\sum_{q=0}^{q=k_{0}-1}(t+q) l_{k_{0}-q}^{\prime} f(s, t, q)\right) \\
& =\sum(t+q)\left(t+k_{0}\right) \cdots\left(t+k_{r-1}\right) l_{k_{0}-q}^{\prime} l_{d_{1}}^{\prime} \cdots l_{d_{r}}^{\prime} f(s, t, q),
\end{aligned}
$$

the sum being now taken for all integer $q$ and $k_{i}$ such that:

$$
0 \leqq q<k_{0}<k_{1}<\cdots<k_{r-1}<k_{r}=k \text { and } d_{i}=k_{i}-k_{i-1} \text {. }
$$

Changing the notation and writing $k_{0}$ for $q, k_{1}$ for $k_{0}$ and, generally $k_{i+1}$ for $k_{i}$ we obtain $\left(4.10^{\prime}\right)$ and $\left(4.10^{\prime \prime}\right)$ for $r+1$.

If now in $\left(4.10^{\prime}\right)$ we put $s=0$ and use (4.3), we see that in this case only the terms with $k_{0}=0$ remain so that:

$$
\frac{\partial^{r}}{\partial s^{r}} f(0, t, k)=\sum\left(t+k_{0}\right)\left(t+k_{1}\right) \cdots\left(t+k_{r-1}\right) l_{d_{1}}^{\prime} l_{d_{2}}^{\prime} \cdots l_{d_{r}}^{\prime}
$$

the sum being taken for all integer $k_{i}$ such that:

$$
0=k_{0}<k_{1}<\cdots<k_{r-1}<k_{r}=k \text { and } d_{i}=k_{i}-k_{i-1} \text {. }
$$


Using the fact that

$$
f(s, t, k)=\sum_{r=0}^{r=+\infty} \frac{1}{r !} \frac{\partial^{r}}{\partial s^{r}} f(0, t, k) s^{r}
$$

and formulas (4.3) and (4.11) we obtain formulas $\left(4.2^{\prime}\right)$ to $\left(4.2^{\prime \prime \prime \prime}\right)$ with $l_{j}^{\prime}$ written for $l_{j}$.

It remains to show that the sequence $\left\{l_{j}^{\prime}\right\}$ of (4.7) is identical to the sequence $\left\{l_{j}\right\}$ introduced in (4.1). This however, results from (4.2) in the case $t=1$ and $s=1$. We find that the coefficients of the power series expansion of $F(1, z)$ are actually given by formulas $\left(4.1^{\prime}\right)$ and $\left(4.1^{\prime \prime}\right)$ with $l_{j}^{\prime}$ written for $l_{j}$. As these formulas determine uniquely the $l_{j}$ in terms of the coefficients of the power series expansion of $F(z)=F(1, z)$, the two sequences determined by (4.1) and by (4.7) are identical and $l_{j}^{\prime}=l_{j}$ for all $j$, which concludes the proof of our main theorem.

In the particular case when $t$ is an integer the numbers $t+k_{i}$ in (4.2) are also integers. Writing in this particular case:

$$
t=m \quad \text { and } \quad f(s, t, k)=f_{t k}(s)
$$

Theorem I becomes:

THEOREM II. Let $m$ be an integer and let $s \in S$ be any complex number for which $F(s, z)$ is defined. Let:

$$
(F(s, z))^{m}=\sum_{k=m}^{k=+\infty} f_{m \cdot k}(s) z^{k}
$$

Then:

$$
f_{m, m}(s)=1,
$$

and:

$$
f_{m, k}(s)=\sum \frac{s^{r}}{r !} k_{0} k_{1} \cdots k_{r-1} l_{d_{1}} l_{d_{2}} \cdots l_{d_{r}}, \quad \quad(k>m),
$$

the sum being taken for all integer $r$ and all integer $k_{i}$ such that:

$$
m=k_{0}<k_{1}<\cdots<k_{r-1}<k_{r}=k \text { and } d_{i}=k_{i}-k_{i-1} \text {. }
$$

Using the fact that:

$$
\log \frac{F(s, z)}{z}=\lim _{t \rightarrow 0} \frac{1}{t}\left(\frac{F(s, z)^{t}}{z^{t}}-1\right),
$$

we can deduce from Theorem I the following additional result:

THEOREM III. Let $s \in S$ be any complex number for which $F(s, z)$ is defined and let: 


$$
\log \frac{F(s, z)}{z}={ }_{k=1}^{k=+\infty} \lambda_{k}(s) z^{k}
$$

then:

$$
\lambda_{k}(s)=\sum \frac{s^{r}}{r !} k_{1} k_{2} \cdots k_{r-1} l_{d_{1}} l_{d_{2}} \cdots l_{d_{r}}
$$

the sum being taken for all integer $r$ and all integer $k_{i}$ such that:

$$
0=k_{0}<k_{1}<\cdots<k_{r-1}<k_{r}=k \text { and } d_{i}=k_{i}-k_{i-1} .
$$

\section{GRUNSKY'S COEFFICIENTS FOR SCHLICHT FUNCTIONS}

We now proceed to establish the following theorem:

THEOREM IV. Consider the function $F(z) \in \Omega$ and put:

$$
\log \frac{F(w)-F(z)}{w-z}=\log \frac{F(w)}{w}+\log \frac{F(z)}{z}+\sum_{m, n=1}^{m \cdot n=+\infty} g_{m, n} w^{m} z^{n}
$$

then:

$$
g_{m n}=\sum \frac{1}{r !}\left(\begin{array}{c}
r-1 \\
v
\end{array}\right)\left|k_{1} k_{2} \cdots k_{r-1}\right| l_{d_{1}} l_{d_{2}} \cdots l_{d},
$$

the sum being taken for all integer $r$ and all integer $k_{i}$ such that:

$$
-m=k_{0}<k_{1}<k_{2}<\cdots<k_{r-1}<k_{r}=n \text { and } d_{i}=k_{i}-k_{i-1},
$$

$v$ being the number of negative $k_{i}$ with $i>0$ (that is, $k_{v}<0<k_{v+1}$ ).

Or, alternatively:

$$
g_{m, n}=\sum \frac{p q}{p+q-1} \cdot \frac{1}{p !} k_{1} k_{2} \cdots k_{p-1} l_{d_{2}} \cdots l_{d_{p}} \cdot \frac{1}{q !} k_{1}^{\prime} k_{2} \cdots k_{q-1}^{\prime} l_{d_{2}}^{\prime} \cdots l_{d_{a}}^{\prime} l_{k_{1}+k_{1}}^{\prime}
$$

the sum being taken for all integer $p$ and $q$ and all integer $k_{i}$ and $k_{i}^{\prime}$ such that:

$$
0<k_{1}<k_{2}<\cdots<k_{p-1}<k_{p}=m, 0<k_{1}^{\prime}<k_{2}^{\prime}<\cdots<k_{q-1}^{\prime}<k_{q}^{\prime}=n,
$$

and:

$$
d_{i}=k_{i}-k_{i-1}, \quad d_{i}^{\prime}=k_{i}^{\prime}-k_{i-1}^{\prime} .
$$

Proof. The function $\log ((F(w)-F(z)) /(w-z))$, considered as a function of $w$ and $z$, has singularities only at the singularities of $F(w)$ and of $F(z)$ or when the argument of the logarithm is infinite or is zero. Let $\rho$ be the radius of schlichtness of $F(z)$. For $|z|<\rho$ and $|w|<\rho$ we have $F(w)-F(z)=0$ only when $w-z=0$. In that case, however, $(F(w)-F(z)) /(w-z)$ becomes equal to $F^{\prime}(w)$ and, because of the schlichtness condition, $F^{\prime}(w) \neq 0$ and $F^{\prime}(w) \neq \infty$ for $|w|<\rho$. Thus $\log ((F(w)-F(z)) /(w-z))$ is analytic in $w$ and in $z$ in some neighborhood of $z=0$ and $w=0$ and is represented there by an absolutely convergent double power series, so that: 
$\log \frac{F(w)-F(z)}{w-z}=\sum_{m, n=0}^{m, n=+\infty} g_{m, n} w^{m} z^{n}=\sum_{m=0}^{m=+\infty} g_{m, 0} w^{m}+\sum_{n=0}^{n=+\infty} g_{0, n} z^{n}+\sum_{m, n=1}^{m, n=+\infty} g_{m, n} w^{m} z^{n}$.

Putting first $z=0$ and then $w=0$, we easily see that the sums of the first two power series are respectively $\log F(w) / w$ and $\log F(z) / z$. Therefore:

$$
\log \frac{F(w)-F(z)}{w-z}=\log \frac{F(w)}{w}+\log \frac{F(z)}{z}+\sum_{m \cdot n=1}^{m \cdot n=+\infty} g_{m \cdot n} w^{m} z^{n} .
$$

Rearranging, we obtain:

$$
\sum_{m, n=1}^{m, n} g_{m, n} w^{m} z^{n}=\log \left[1-\frac{F(z)}{F(w)}\right]-\log \left(1-\frac{z}{w}\right)-\log \frac{F(z)}{z} .
$$

Limiting ourselves to values of $w$ and $z$ such that $|w|>|z|$ and $|F(w)|>|F(z)|$ we find:

$$
-\log \left(1-\frac{z}{w}\right)=\sum_{q=1}^{q=+\infty} \frac{1}{q} w^{-q} z^{q}
$$

and, using (2.1):

$$
\begin{aligned}
\log \left(1-\frac{F(z)}{F(w)}\right) & =\sum_{p=1}^{p=+\infty} \frac{-1}{p}[F(z)]^{p}[F(w)]^{-p} \\
& =\sum_{p=1}^{p=+\infty} \frac{-1}{p}\left[\sum_{n=p}^{n=+\infty} f_{p . n} z^{n}\right]\left[\sum_{m=-p}^{m=+\infty} f_{-p, m} w^{m}\right] \\
& =\sum_{n=1}^{n=+\infty} \sum_{m=-n}^{m=+\infty}\left(\sum_{p=1}^{p=n} \frac{-1}{p} f_{p n} f_{-p, m}\right) w^{m} z^{n} .
\end{aligned}
$$

(The change in the order of summation is permissible because we are dealing with absolutely convergent series.)

Substituting into (5.3) we obtain:

$$
\begin{aligned}
\sum_{m, n=1}^{m, n=+\infty} g_{m . n} w^{m} z^{n}= & \sum_{n=1}^{n=+\infty} \sum_{m=-n}^{m=+\infty}\left(\sum_{p=1}^{p=n} \frac{-1}{p} f_{p . n} f_{-p . m}\right) w^{m} z^{n} \\
& +\sum_{q=1}^{q=+\infty} w^{-q} z^{q}-\log \frac{F(z)}{z}
\end{aligned}
$$

Comparing the coefficients of $w^{m} z^{n}$ on both sides of (5.4) we get:

$$
\left.g_{m, n}=\sum_{p=1}^{p=n} \frac{-1}{p} f_{p, n} f_{-p m} \quad \text { (for } m, n>1\right) .
$$

Note that the terms in the zero or negative powers of $w$ on the right side of (5.4) have to disappear because there are no such terms on the left side. This implies that for $m<0$ and $n>0$ we have: 


$$
\left.\sum_{p=1}^{p=n} \frac{1}{p} f_{p, n} f_{-p, m}=\frac{1}{n} \delta_{-m, n} \quad \text { (for } m<0<n\right)
$$

and:

$$
\sum_{=1}^{p=n} \frac{1}{p} f_{p, n} f_{-p, 0}=-\phi_{n} \text { with } \sum_{n=1}^{n=+\infty} \phi_{n} z^{n}=\log \frac{F(z)}{z}(\text { for } m=0 \text { and } 0<n)
$$

Using a theorem on the representation of inverse functions [15;7], formulas (5.6) and (5.7) can be proven directly and be used to establish (5.5) without invoking the existence of a radius of schlichtness. This was done in [8].

It now remains to deduce (5.1) from (5.5). By Theorem II (written for the case $s=1)$ we have:

$$
f_{-p, m}=\sum \frac{1}{a !} \lambda_{0} \lambda_{1} \cdots \lambda_{a-1} l_{\gamma_{1}} l_{\gamma_{2}} \cdots l_{\gamma_{\alpha}},
$$

the sum being taken for all integer $a$ and all integer $\lambda_{i}$ such that:

$$
-p=\lambda_{0}<\lambda_{1}<\cdots<\lambda_{a-1}<\lambda_{a}=m \text { and } \gamma_{i}=\lambda_{i}-\lambda_{i-1},
$$

and:

$$
f_{p, n}=\Sigma \frac{1}{b !} \mu_{0} \mu_{1} \cdots \mu_{b-1} l_{\delta_{1}} l_{\delta_{2}} \cdots l_{\delta_{b}},
$$

the sum being taken for all integer $b$ and all integer $\mu_{i}$ such that:

$$
p=\mu_{0}<\mu_{1}<\cdots<\mu_{b-1}<\mu_{b}=n \text { and } \delta_{i}=\mu_{i}-\mu_{i-1} .
$$

We now note that $-\lambda_{0}=\mu_{0}=p$. Putting:

$$
\lambda_{i}=-k_{a-i} \text { and } \mu_{j}=k_{a+j} \quad(\text { for } i=0,1, \cdots, a \text { and } j=0,1, \cdots, b),
$$

and writing:

$$
a+b=r,
$$

we can combine (5.8) with (5.9) and obtain the following preliminary result:

$$
\frac{-1}{p} f_{-p, m} f_{p, n}=\sum \frac{1}{a !} \frac{1}{(r-a) !}(-1)^{a+1} k_{1} k_{2} \cdots k_{r-1} l_{d_{1}} l_{d_{2}} \cdots l_{d_{r}},
$$

the sum being taken for all integer $r$, all integer $a$ and all integer $k_{i}$ such that:

$$
-m=k_{0}<k_{1}<\cdots<k_{r-1}<k_{r}=n \text { and } d_{i}=k_{i}-k_{i-1} .
$$

If we now proceed to sum terms like (5.10) for $p=1,2, \cdots, n$ there will clearly appear several terms containing the same product:

$$
k_{1} k_{2} \cdots k_{r-1} l_{d_{1}} l_{d_{2}} \cdots l_{d_{r}} \text {, }
$$


but this product will appear each time with a different coefficient $(-1)^{a+1} / a !(r-a)$ !. The values of $p$ for which the product (5.11) will appear are $p=k_{v+1}, k_{v+2}, \cdots, k_{r}$ where $v$ is the number of the $k_{i}$ with $i>0$ which are negative (that is $\left.k_{1}<k_{2}<\cdots<k_{v}<0<k_{v+1}<\cdots<k_{r}\right)$. Indeed $p$ is positive and it is one of the $k_{i}$ (because of the condition $p=-\lambda_{0}=\mu_{0}=k_{a}$ ). Thus, in the total sum, the product (5.11) will appear with the coefficient:

$$
\sum_{a=v+1}^{a=r} \frac{(-1)^{a+1}}{a !(a-r) !}=(-1)^{v} \frac{1}{r !}\left(\begin{array}{c}
r-1 \\
v
\end{array}\right) .
$$

Noting that exactly $v$ of the $k_{i}$ in the product (5.11) have the minus sign, we see that the sign of the product $(5.11)$ is also $(-1)^{\nu}$. Thus the contribution of the terms containing the product (5.11) to the sum (5.5) is:

$$
\frac{1}{r !}\left(\begin{array}{c}
r-1 \\
v
\end{array}\right)\left|k_{1} k_{2} \cdots k_{r-1}\right| l_{d_{1}} l_{d_{2}} \cdots l_{d_{r}}
$$

which proves (5.1) for values of $w$ and $z$ for which $|w|>|z|$ and $|F(w)|>|F(z)|$, so that both sides of (5.1) represent the same analytic function, which proves (5.1).

Equations (5.2) are equations (5.1) rewritten with $p=v+1, q=r-v$, $k_{i}=\left|k_{v-i+1}\right|(i=1,2, \cdots, p)$ and $k_{i}^{\prime}=k_{v+j}(j=1,2, \cdots, q)$.

\section{A THEOREM ON ANALYTIC ITERATION}

In [3] it was proven that if the series (3.5) for $L(z)$ has a positive radius of convergence, then the corresponding function $F(z)$ has an analytic iterate. The proof uses existence theorems from the theory of differential equations. We are now in a position to give a direct proof. Indeed, if the series $L(z)=\sum_{k=1}^{k=+\infty} l_{k} z^{k+1}$ has a positive radius of convergence then there exist a number $\lambda>0$ and a number $A>0$ such that:

$$
\left|l_{k}\right|<A \lambda^{k}
$$

Taking $m=1$ in (4.13) we see that the coefficient of $z^{k}$ in the power series expansion of $F(s, z)$ is smaller than:

$$
\sum \frac{|s|^{r}}{r !} k_{1} k_{2} \cdots k_{r-1} A^{r} \lambda^{k}
$$

the sum being taken for all integer $r$ and all integer $k_{i}$ such that:

$$
1<k_{1}<k_{2}<\cdots<k_{r-1}<k+1 \text {. }
$$

The number of terms in the sum $\left(6.2^{\prime}\right)$ is $2^{k-1}$ as each one of the numbers between 2 and $k$ can be taken as a $k_{i}$ or not. Each of these terms is less or equal to: 


$$
T=\frac{1}{r !} \frac{k !}{(k-r) !}(A|s|)^{r} \lambda .
$$

The largest value of $T$ in (6.3) is obtained for:

$$
r=\frac{A|s|}{1+A|s|} k .
$$

Using Stirling's theorem we find that $T$ in (6.3) then takes a value of the order of:

$$
T^{*}=\frac{c}{\sqrt{ } k} B^{k},
$$

where $B$ is a positive number given by:

$$
B=\frac{\lambda(A|s|)^{A|s|}}{1+A|s|} .
$$

Therefore the coefficient $f_{k}(s)$ of $z^{k}$ in the expansion $F(s, z)$ is of the order of:

$$
\frac{c}{2 \sqrt{ } k}(2 B)^{k}
$$

and the radius of convergence $\rho$ of $F(s, z)$ satisfies the inequality:

$$
\rho \geqq \frac{1}{2 B}>0,
$$

which proves the theorem.

$$
\text { 7. THE CASE } l_{k}=M \rho^{-k}
$$

We shall consider the function $F(z)$ whose $L$-function is, $M$ and $\rho$ being $>0$ :

$$
L(z)=\frac{\frac{M}{\rho} z^{2}}{1-\frac{z}{\rho}}=\sum_{k=1}^{k=+\infty} M \rho^{-k} z^{k+1} \text {, that is: } l_{k}=M \rho^{-k} \text {. }
$$

The properties established for this function can be used to obtain some less precise information about a larger family of functions $\in \Omega$ for which the $L$-sequence $\left\{l_{k}\right\}$ has only real, non-negative terms $l_{k}$ such that:

$$
0 \leqq l_{k} \leqq M \rho^{-k},
$$$$
(M, \rho>0) \text {. }
$$

This information can, in turn, be used to obtain still less precise results concerning the whole family of functions $F(z) \in \Omega$ which have an analytic iterate. By [3, Theorem 1], this is the family of the functions $F(z) \in \Omega$ whose $L$-sequence $\left\{l_{k}\right\}$ is such that there exist two numbers $M>0$ and $\rho>0$ such that:

$$
\iota_{k}<M \rho^{-k}, \quad(k=1,2, \cdots) \text {. }
$$


These generalizations will, however, not be attempted here.

By (7.1) we have here:

Put:

$$
L(z)=\frac{\frac{M}{\rho} z^{2}}{1-\frac{M}{\rho} z} .
$$

$$
w=F(s, z)
$$

Equation (3.12) becomes:

$$
\frac{1-\frac{M}{\rho} w}{\frac{M}{\rho} w^{2}} d w=\frac{1-\frac{M}{\rho} z}{\frac{M}{\rho} z^{2}} d z
$$

Integrating we find:

$$
-\frac{1}{w}-\frac{1}{\rho} \log w=c-\frac{1}{z}-\frac{1}{\rho} \log z .
$$

The integration constant $c$ should be chosen in such a manner that equation (7.6) be satisfied by an expansion whose first terms are:

$$
w=F(s, z)=z+s \frac{M}{\rho} z^{2}+\cdots,
$$

(this will ensure that $l_{1}=M / \rho$ ). Substituting (7.7) into (7.6), we easily find that:

$$
c=\frac{M}{\rho} \text {. }
$$

Using this value for $c$ and multiplying by $(-\rho)$ equation (7.6) becomes:

$$
\frac{\rho}{w}+\log w=-M s+\frac{\rho}{z}+\log z
$$

and, in particular, for $s=1$ we have:

$$
\frac{\rho}{w}+\log w=-M+\frac{\rho}{z}+\log z \text { with } w=F(z) .
$$

It is easy to show that the function $\rho / z+\log z$ has no exceptional values in the sense of Picard (that is that this function takes all complex values at least once). The solutions $w=\psi(z)$ of an equation of the type $\phi(w)=\Psi(z)$, where the analytical functions $\phi$ and $\Psi$ are devoid of exceptional values, may have singularities only for values of $z$ which are poles or singularities of the function $\Psi(z)$ or zeros of $\Psi^{\prime}(z)$ and for values of $w$ which are poles or singularities of the function $\phi(w)$ or zeros of $\phi^{\prime}(w)$. Hence, in the case of equation (7.10), its solution $w=F(z)$ may have singularities only for

$$
z=0, z=\infty, w=0 \text { or } w=\infty
$$


or, as:

for:

$$
\frac{d}{d w}\left(\frac{n}{w}+\log w\right)=\frac{w-\rho}{w^{2}} \text { and } \frac{d}{d z}\left(-M+\frac{\rho}{z}+\log z\right)=\frac{z-\rho}{z^{2}}
$$

$$
z=\rho \quad \text { or } \quad w=\rho .
$$

We know [3] that there exists a branch of the function $w=F(z)$ which for $z=0$ takes the value $w=0$ and which is analytic at this point. The singularity of the function $F(z)$ with the smallest $|z|$ is hence either at the point $z=\rho$ or at the point for which $w=\rho$. As all the $l_{k}$ are $>0$, by (4.1) all the $f_{k}$ are positive and hence the first singularity of $F(z)$ occurs at a point $z=r$ of the positive real half axis in the $z$-plane. We have either $r=\rho$ or $0<r<\rho$. In the latter case we have to have $w=\rho$ for $z=r$ or, by (7.10):

$$
1+\log \rho=-M+\frac{\rho}{r}+\log r \text {. }
$$

We shall show that, for given $M$ and $\rho>0$, equation (7.12) is always satisfied by just one value of $r$ such that:

$$
0<r<\rho .
$$

Indeed put:

$$
\frac{r}{\rho}=x
$$

Equation (7.12) becomes:

$$
M+1=\frac{1}{x}+\log x
$$

The curve $y=1 / x+\log x$ for $0<x$ starts from $+\infty$, has a minimum for $x=1$ for which $y=1$ and increases again to $+\infty$. Therefore $y$ takes every real value $>1$ just once for $0<x<1$, that is, by (7.14), for $0<r<\rho$. It follows that the first singularity of $F(z)$ is attained at a point where $w=\rho$ and that there $z=r$, where $r$ satisfies equation (7.12) and inequality (7.13).

The radius of convergence $\rho_{F}$ of the series $\left(4.1^{\prime}\right)$ of $F(z)$ is therefore:

$$
\rho_{F}=r \text {. }
$$

Let $\rho_{\Lambda}$ be the radius of convergence of the series $\left(1.2^{\prime}\right)$ for $\log (F(z) / z)$ and let us rewrite formulas (4.1) for $f_{k+1}$ (instead of $f_{k}$ ) and compare them with the formulas (4.15) for $\lambda_{k}$ for the case $s=1$. It is clear that exactly the same products $l_{d_{1}} \cdots l_{d_{r}}$ appear now in both formulas but in the sum for $f_{k+1}$ they appear with coefficients $k_{1} \cdots k_{r-1}$ in which the $k_{i}$ are larger by one than the $k_{i}$ appearing in the coefficients of the same products in the sum for $\lambda_{k}$. Hence, if: 


$$
l_{i} \geqq 0
$$$$
(i=1,2, \cdots) \text {, }
$$

(which certainly holds if $l_{i}=M \rho^{-i}$ ), then:

$$
\lambda_{k} \leqq f_{k+1} \text {. }
$$

It follows that, in this case:

$$
\rho_{\Lambda} \geqq \rho_{F} .
$$

But, from general considerations of analyticity:

$$
\rho_{\Lambda} \leqq \rho_{F}
$$

Therefore:

$$
\rho_{\Lambda}=\rho_{F} .
$$

Let $\rho_{S}$ be the radius of schlichtness of $F(z)$. By (1.3) we have:

$$
\rho_{S}^{-1}=\limsup \left|g_{m, n}\right|^{1 /(m+n)} \text {. }
$$

For $l_{i}=M \rho^{-i}$ we have from $\left(5.2^{\prime}\right)$ and $\left(5.2^{\prime \prime}\right)$ :

$$
\begin{aligned}
g_{m, n} & =\sum \frac{p q}{p+q-1} \frac{1}{p !} k_{1} \cdots k_{p-1} \cdot \frac{1}{q !} k_{1}^{\prime} \cdots k_{q-1}^{\prime} M^{p+q-1} \rho^{-(m+n)} \\
& \leqq \frac{m n}{M} \sum \frac{1}{p !} k_{1} \cdots k_{p-1} M^{p} \rho^{-m} \frac{1}{q !} k_{1}^{\prime} \cdots k_{q-1}^{\prime} M^{q} \rho^{-n} \\
& =\frac{m n}{M} f_{m} f_{n} .
\end{aligned}
$$

We have here noted that $p q /(q+q-1)<m n$. Similarly by noting that $p q /(p+q-1)>1 /(m+n)$ we find that:

$$
g_{m, n}>\frac{1}{(m+n) M} f_{m} f_{n} .
$$

From (7.17), (7.23) and (7.24) we easily get:

$$
\rho_{S}=\rho_{F} .
$$

Thus we have proved:

THEOREM V. Let $F(z) \in \Omega$ be the function for which:

$$
l_{k}=M \rho^{-k} .
$$

Then the radius of analyticity $\rho_{F}$ of $F(z)$, the radius of analyticity $\rho_{\Lambda}$ of $\log (F(z) / z)$ and the radius of schlichtness $\rho_{S}$ of $F(z)$ are all equal and their common value $r$ satisfies the equation: 


$$
1+M=\frac{\rho}{r}-\log \frac{\rho}{r} \text { with } r<\rho .
$$

In particular if $1+M=\rho-\log \rho$, that is if:

$$
l_{k}=(\rho-1-\log \rho) \rho^{-k},
$$

then:

$$
r=\rho_{F}=\rho_{\Lambda}=\rho_{S}=1 .
$$

\section{FURTHER POSSIBLE DEVELOPMENTS}

The methods used in this paper constitute a mathematical tool for the possible further study of various families af analytic functions. Three such families could be mentioned:

(1). The family of the functions $F(z) \in \Omega$ which have an analytic iterate. By the preceding paragraph and by [3] this family is identical to the family for which there exist $A>0$ and $\lambda>0$ such that

$$
\left|l_{k}\right| \leqq A \lambda^{k} .
$$

It is interesting to find data on the configuration space of $\left(f_{2}, f_{3}, \cdots\right)$ for these functions.

(2). The family of functions $F(z) \in \Omega$ which have an analytic iterate and such that $F(s, z)$ is analytic in the unit circle for all $|s| \leqq 1$. This is a sub-family of (1). It can be shown that it is obtained by limiting $A$ in (7.1):

$$
A \leqq A_{\lambda} .
$$

(3). The family of functions $F(z) \in \Omega$ are such that both they and their inverses $F(-1, z)$ are schlicht in the unit circle. Such a study has been made by $M$. Lewin and some as yet unpublished interesting results have been obtained [11].

(4). From a different point of view, the present paper may be expanded by replacing the representation of functions by matrices by a more general representation: that by two parameter integral transforms.

A double integral transform is defined as follows:

Let $B(x, z)$ be defined for real $x$ and complex $z$. Let $F(z)$ be an analytic function (not necessarily $\in \Omega$ ). Then a function $f(x, y)(x, y$ real) will be a two parameter integral transform of $F(z)$ if:

$$
B(x, F(z))=\int_{y=-\infty}^{y=+\infty} B(y, z) d f(x, y) \quad(x=\text { const. })
$$

(It may be noted that if we chose in particular:

$$
B(x, z)=z^{[x]},
$$


then $f(x, y)$ becomes a double step function and can be identified with the representation matrix of $F(z)$. Representation matrices are thus a special case of two parameter integral transforms.)

The effectiveness of the representation of functions by matrices resulted from the fact that one of its lines $-f_{1, k}$-defines the whole matrix so that the knowledge of all the $f_{m, k}$ yields superfluous information. But the inner relations which exist between the $f_{m, k}$ permit great elasticity in writing down formulas, yielding such explicit results as our Theorems I-IV. An even greater elasticity should be achievable by using two parameter integral transforms where the base function $B(x, z)$ is at our disposal and can be chosen ad hoc for each problem.

\section{ACKNOWLEDGEMENT}

The author's thanks are due to A. Gutwirth who pointed out the connection and the differences between a joint theorem of the author with P. Erdös [3] and theorem of D. Montgomery and S. Bochner [2], and also to B. Schwartz, who read through parts of this paper, and, more particularly, to J. A. Jenkins who pointed out an error of principle, which has been corrected.

\section{BIBLIOGRAPHY}

1. I. N. Baker, Zusammensetzung ganzer Funktionen, Math. Z. 69 (1958), 121-163.

2. S. Bochner and D. Montgomery, Groups of differentiable and real or complex analytic transformations, Ann. of Math. (2) 46 (1945), 685-694.

3. P. Erdös and E. Jabotinsky, On analytic iteration, J. Analyse Math. 8 (1960-1961), 361-376.

4. H. Grunsky, Koeffizientenbedingungen für schlicht abbildende meromorphe Funktionen, Math. Z. 45 (1939), 29-61.

5. J. Hadamard, Two works on iteration, Bull. Amer. Math. Soc. 50 (1944), 67-75.

6. E. Jabotinsky, Sur la répresentation de la composition de fonctions par un produit de matrices, C. R. Acad. Sci. Paris 224 (1947), 323-324.

7. —_, Sur les fonctions inverses, C. R. Acad. Sci. Paris 229 (1949), 508-509.

8. - - Representation of functions by matrices, application to Faber polynomials, Proc. Amer. Math. Soc. 4 (1953), 546-553.

9. - Iteration, Thesis. The Hebrew University, Jerusalem, Israel, 1955, pp. 1-120.

10. — On iterational invariants, Technion, Israel Inst. of Tech. Publ. 6 (1954-55) pp. $68-80$.

11. M. Lewin, Analytic iteration of certain analytic functions, M. Sc. Thesis, Technion, Israel Inst. of Tech., Haifa, 1960.

12. J. A. Jenkins, Univalent functions and conformal mapping, Springer, Berlin, (1958).

13. M. Schiffer, Faber polynomials in the theory of univalent functions, Bull. Amer. Math. Soc. 54 (1948), 503-517.

14. I. Schur, On Faber polynomials, Amer. J. Math. 67 (1945), 33-41.

15. G. Szekeres, Regular iteration of real and complex functions, Acta. Math. 100 (1960), 203-258.

The Technion, Israel Institute of Technology, HAIFA, IsRaEL 